\title{
Editorial
}

\section{First science highlights from SRG/eROSITA}

The eROSITA X-ray telescope on the Spectrum-Roentgen-Gamma (SRG) space mission was launched on July 13, 2019. It is conducting a full-sky survey that will be 25 times more sensitive than the ROSAT All-Sky Survey in the $0.2-2.4$ keV X-ray band and will provide the first true imaging survey of the $2.4-8 \mathrm{keV}$ sky. The 12 papers of this special issue describe the instrument, its performance in pre-launch tests and in space, and some of its early observations. These observations confirm that the performance of the instrument will fulfill its scientific promise. At the same time, their results provide a teaser of what is to come on a broad range of astronomy topics, including large supernova remnants in our Galaxy, filaments between galaxy clusters, and X-ray binaries and active galactic nuclei.

Sergio Campana, Françoise Combes, David Elbaz, Thierry Forveille, Rubina Kotak, Laura Pentericci, and Steve Shore Astronomy \& Astrophysics Editors 